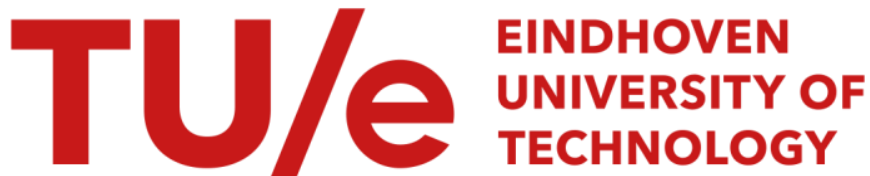

\section{Finite thermal conductivity in 1D lattices}

\section{Citation for published version (APA):}

Giardinà, C., Livi, R., Politi, A., \& Vassalli, M. (2000). Finite thermal conductivity in 1D lattices. Physical Review Letters, 84(10), 2144-2147. https://doi.org/10.1103/PhysRevLett.84.2144

DOI:

10.1103/PhysRevLett.84.2144

Document status and date:

Published: 01/01/2000

\section{Document Version:}

Publisher's PDF, also known as Version of Record (includes final page, issue and volume numbers)

\section{Please check the document version of this publication:}

- A submitted manuscript is the version of the article upon submission and before peer-review. There can be important differences between the submitted version and the official published version of record. People interested in the research are advised to contact the author for the final version of the publication, or visit the $\mathrm{DOI}$ to the publisher's website.

- The final author version and the galley proof are versions of the publication after peer review.

- The final published version features the final layout of the paper including the volume, issue and page numbers.

Link to publication

\section{General rights}

Copyright and moral rights for the publications made accessible in the public portal are retained by the authors and/or other copyright owners and it is a condition of accessing publications that users recognise and abide by the legal requirements associated with these rights.

- Users may download and print one copy of any publication from the public portal for the purpose of private study or research.

- You may not further distribute the material or use it for any profit-making activity or commercial gain

- You may freely distribute the URL identifying the publication in the public portal.

If the publication is distributed under the terms of Article 25fa of the Dutch Copyright Act, indicated by the "Taverne" license above, please follow below link for the End User Agreement:

www.tue.nl/taverne

Take down policy

If you believe that this document breaches copyright please contact us at:

openaccess@tue.nl

providing details and we will investigate your claim. 


\title{
Finite Thermal Conductivity in 1D Lattices
}

\author{
C. Giardiná, ${ }^{1,2}$ R. Livi, ${ }^{3,4}$ A. Politi, ${ }^{5,4}$ and M. Vassalli ${ }^{3}$ \\ ${ }^{1}$ Dipartimento di Fisica, via Irnerio 46, 40126 Bologna, Italy \\ ${ }^{2}$ Istituto Nazionale di Fisica Nucleare, Sezione di Bologna, Bologna, Italy \\ ${ }^{3}$ Dipartimento di Fisica, L.go E. Fermi 2, 50125 Firenze, Italy \\ ${ }^{4}$ Istituto Nazionale di Fisica della Materia, Unità di Firenze, Firenze, Italy \\ ${ }^{5}$ Istituto Nazionale di Ottica Applicata, L.go E. Fermi 6, 50125 Firenze, Italy
}

(Received 18 October 1999)

\begin{abstract}
We discuss the thermal conductivity of a chain of coupled rotators, showing that it is the first example of a 1D nonlinear lattice exhibiting normal transport properties in the absence of an on-site potential. Numerical estimates obtained by simulating a chain in contact with two thermal baths at different temperatures are found to be consistent with those based on linear response theory. The dynamics of the Fourier modes provides direct evidence of energy diffusion. The finiteness of the conductivity is traced back to the occurrence of phase jumps. Our conclusions are confirmed by the analysis of two variants of this model.
\end{abstract}

PACS numbers: 44.10. $+\mathrm{i}$, 05.45.Jn, 05.60. $-\mathrm{k}$, 05.70.Ln

The understanding of heat conduction in insulating solids is a long-standing problem even in the simple 1D context. The typical Hamiltonian used to model this class of systems is

$$
H=\sum_{i=1}^{N}\left[\frac{p_{i}^{2}}{2 m_{i}}+V\left(q_{i+1}-q_{i}\right)+U\left(q_{i}\right)\right],
$$

where $m_{i}$ represents the mass of the $i$ th particle [1], $V$ is the potential energy of internal forces, and $U$ is an on-site potential representing possible interactions with an external substrate.

Rigorous studies have shown that the thermal conductivity $\kappa$ diverges (in the thermodynamic limit) in homogeneous harmonic chains [2], as well as in any integrable model, since the dynamics can be decomposed in that of independent modes (phonons, solitons) freely propagating along the chain. The addition of (isotopic) disorder leads to a qualitatively different scenario depending on the presence of external forces. Systems interacting with a substrate (SS) behave as insulators (vanishing conductivity) in view of the exponential localization of the normal modes. Conversely, isolated systems (IS) still exhibit anomalous transport properties [3], because the localization length of normal modes diverges in the limit of vanishing wave numbers. In either case, normal transport is not observed, suggesting that the nonlinear character of the microscopic dynamics is a necessary ingredient for the emergence of the Fourier heat-conduction law at the macroscopic level.

In fact, the first convincing numerical evidence of a finite conductivity has been provided by the study of a chaotic system: the ding-a-ling model [4], i.e., an SS system where a set of particles harmonically anchored to an external periodic lattice alternates with free particles, having elastic interactions with them. The same conclusion has been later confirmed by the study of the ding-dong model [5], a modification of the previous system, where the free particles have been removed, while allowing elastic scattering between the remaining ones.

On the other hand, the recent study of the more realistic Fermi-Pasta-Ulam (FPU) $\beta$ model $\left[V(x)=x^{2} / 2+\right.$ $\left.x^{4} / 4, U=0\right]$ has revealed a power-law divergence of the thermal conductivity as $\kappa \sim N^{2 / 5}$ [6] (where $N$ is the chain length [7]). Such an anomalous behavior has been explained by invoking the self-consistent mode coupling theory [8] in the description of the effective evolution of long-wavelength modes [9].

The apparent discrepancy with the previous results has stimulated the numerical study of various models in the attempt of identifying general rules. As a result, it has been found that, in analogy with linear disordered systems, the presence of an on-site potential plays a crucial role in determining the qualitative behavior of a physical system. In fact, all ISs discussed in the literature have revealed an anomalous behavior of the thermal conductivity (including, e.g., the diatomic Toda lattice [10]). Conversely, all SSs (including the Frenkel-Kontorova model studied in Ref. [11]) have been found to be characterized by normal transport properties.

In this Letter, we show that a simple classification scheme based on the presence or absence of an on-site potential cannot alone account for the normal or anomalous character of transport coefficients. In fact, we discuss some 1D models where momentum is conserved and yet the conductivity is finite.

(A) The rotator model. - The simplest example of a classical-spin 1D model with nearest neighbor interactions lies in the class (1) with $V(x)=1-\cos (x)$ and $U=0$. This model can be read also as a chain of $N$ coupled pendula, where the $p_{i}$ 's and the $q_{i}$ 's represent action-angle variables, respectively. It has been extensively studied $[12,13]$ as an example of chaotic dynamical system that becomes integrable both in the small and high energy limits, when it reduces to a harmonic chain and free rotators, 
respectively. In the two integrable limits, the relaxation to equilibrium slows down very rapidly for most of the observables of thermodynamic interest (e.g., the specific heat) [13]. As a consequence, the equivalence between ensemble and time averages is established over accessible time scales only inside a limited interval of the energy density $\varepsilon$. Here we shall discuss heat conduction for values of the energy density corresponding to strongly chaotic behavior (i.e., half of the Lyapunov spectrum is convincingly positive [14]).

(B) Numerical analysis of the thermal conductivity. - The most natural and direct way to determine $\kappa$ consists in simulating a real experiment, by coupling the left and right extrema of the chain with two thermal baths at temperatures $T_{L}>T_{R}$, respectively. In our simulations we have used Nosé-Hoover models of thermostats [15], both because they can be easily implemented (integrating the resulting equations with a standard algorithm) and because of the smaller finite-size effects (due to the unavoidable contact resistance).

With this setting, a nonequilibrium stationary state sets in characterized by a nonvanishing heat flux $J[6,16]$ :

$$
J=\frac{1}{N} \sum_{i} j_{i}=\frac{1}{N} \sum_{i} \frac{p_{i}}{2}\left(f_{i+1}+f_{i}\right)
$$

where $f_{i}=-\partial V\left(q_{i+1}-q_{i}\right) / \partial q_{i}=\sin \left(q_{i+1}-q_{i}\right)$ is the interaction force and $j_{i}$ is the local flux at site $i$. The total heat flux $J$ has to be averaged over a sufficiently long time span to get rid of fluctuations and to ensure the convergence to the stationary regime. This can be tested by monitoring the average heat flux and looking at the scale of its fluctuations. As a result, we have verified that $2 \times 10^{6}$ time units are sufficient to guarantee a few percent of fluctuations in the worst cases.

The thermal conductivity is determined by assuming the Fourier law, i.e., from the relation $J=\kappa \nabla T$, where $\nabla T$ denotes the imposed thermal gradient. The simulations have been performed for $T_{L}=0.55, T_{R}=0.35$, and chain lengths ranging from $N=32$ to 1024 with fixed boundary conditions. The equations of motion have been integrated with a 4th-order Runge-Kutta algorithm and a time step $\Delta t=0.01$. The results, reported in Fig. 1, clearly reveal a convergence to a $\kappa$ value approximately equal to 7 (see the circles). The dotted line represents the best data fit with the function $a+b / N$ : the agreement is very good, showing that finite-size corrections to $\kappa$ are of the order $O(1 / N)$, as it should be expected because of the thermal contacts. However, more important than the numerical value of the conductivity is its finiteness in spite of the momentum conservation.

In order to test independently the correctness of our results, we have performed direct microcanonical simulations, which allow determining the thermal conductivity through the Green-Kubo formula [17],

$$
\kappa=\frac{1}{T^{2}} \int_{0}^{\infty} C_{J}(t) d t
$$

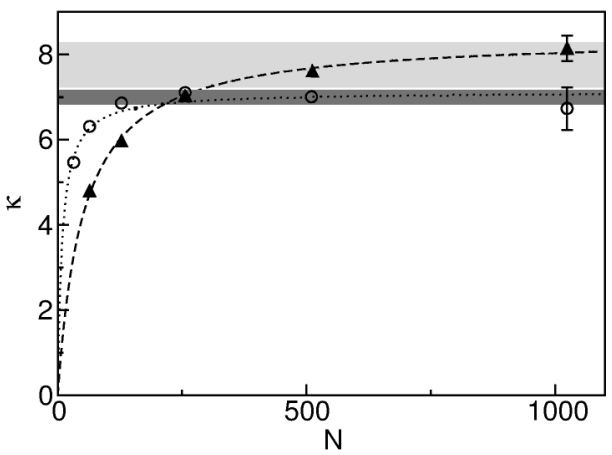

FIG. 1. Conductivity $\kappa$ versus chain length $N$ as obtained from nonequilibrium molecular dynamics. Circles correspond to the rotator model with temperatures $T_{L}=0.55$ and $T_{R}=0.35$; triangles correspond to the double-well potential with $T_{L}=0.04$ and $T_{R}=0.06$. The two lines represent the best fit with the function $a+b / N$. The two shaded regions represent the uncertainty about the conductivity on the basis of the Green-Kubo formula.

where $C_{J}(t)=N\langle J(t) J(0)\rangle$ is the flux autocorrelation function at equilibrium and $T$ is the temperature. A correct application of the above formula requires fixing the energy density $\varepsilon$ in such a way that the kinetic temperature (defined as $T=\left\langle p^{2}\right\rangle$, in agreement with the virial theorem) is close to the average value of the temperature in the previous simulations. The choice $\varepsilon=0.5$ turns out to be reasonable, as it corresponds to $T \approx 0.46$. In the absence of thermal baths, the equations of motion are symplectic, so that we have now preferred to use a sixth order McLachlan-Atela integration scheme [18]. With the adoption of periodic boundary conditions, not only the energy but also the momentum is (in principle) exactly conserved.

The correlation function has been computed by exploiting the Wiener-Khinchin theorem, i.e., by antitransforming the Fourier power spectrum. At variance with the FPU- $\beta$ model studied in Ref. [9], where a power-law convergence to zero was observed, here $C_{J}(t)$ exhibits a clean exponential decay (see Fig. 2). Moreover, the good overlap among the three curves (computed for $N=128,256$, and 1024) indicates that the correlations are independent of the system size already for $N=128$ rotators. The straight line, added below for comparison, corresponds to an exponential decay with a time constant $t_{0}=30$ in our adimensional units.

As a result, the integral of $C_{J}$ manifestly converges to a finite value and is independent of $N$. The gray region in Fig. 1 corresponds to the expected value of $\kappa$ taking into account the statistical uncertainty. There is not only a clear confirmation of a finite conductivity, but the numerical value obtained with this technique is in close agreement with the direct estimates.

(C) Dynamics in the mode space. - In order to clarify the difference between the dynamics of the present model and that of FPU-type chains, we have investigated the evolution of the low-frequency Fourier modes, as they proved 


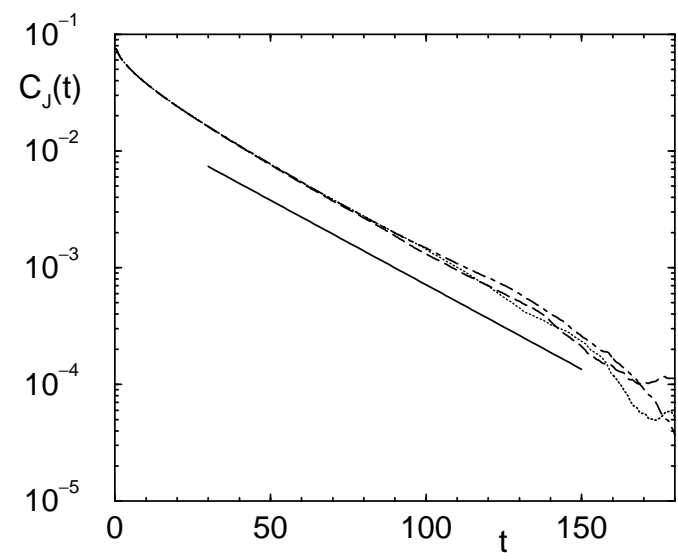

FIG. 2. Correlation function $C_{J}(t)$ of the total flux for 256, 512, and 1024 rotators (dashed, dot-dashed, and dotted lines, respectively) for energy density $\varepsilon=0.5$. The correlation function has been computed by antitransforming the power spectrum computed over more than $10^{4}$ time units and averaged over $10^{3}$ independent trajectories. The solid line, corresponding to the exponential decay $\exp (-t / 30)$, has been added for reference. The deviations observed at large times are due to an insufficient statistics.

to be responsible for the anomalous behavior of such systems. In Fig. 3 we have reported the power spectra of some long-wavelength modes. For the sake of comparison, the same quantities are reported for a diatomic FPU chain that is characterized by an anomalous transport. At variance with the FPU model, in the rotators there is no sharp peak (which is a signal of an effective propagation of correlations [9]). Quite differently, the low-frequency part of the spectrum is described very well by a Lorentzian with half-width $\gamma=D k^{2}(D \approx 4.3)$. This represents an independent proof that energy diffuses, as one expects whenever the Fourier law is established.

(D) Temperature dependence of the thermal conductivity. - The most natural question arising from these results

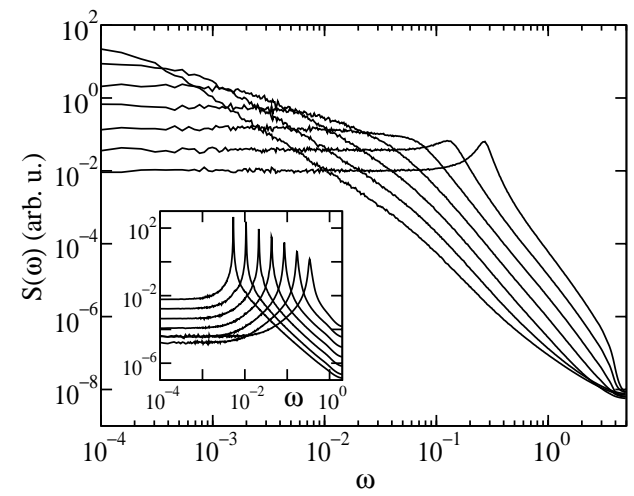

FIG. 3. Power spectra $S(w)$ in arbitrary units of the Fourier modes $1,2,4,8,16,32$, and 64 (curves from left to right in the high frequency region) in a chain of length $N=1024$ of rotators with energy density with energy density 0.5 . The curves result from an average over 1000 independent initial conditions. In the inset, the same modes are reported for a diatomic FPU chain with masses 1, 2 and energy density 8.8 . concerns the reason for the striking difference with other symmetric models such as the FPU- $\beta$ system. As long as each $\left(q_{i+1}-q_{i}\right)$ remains confined to the same valley of the potential, there cannot be any qualitative difference with the models previously studied in the literature. Jumps through the barrier, however, appear to act as localized random kicks that contribute to scattering the low-frequency modes and thus to a finite conductivity. If this intuition is correct, one should find analogies between the temperature dependence of the conductivity and the average escape time from the potential well. To this aim, we have computed $\kappa$ for different temperature values by performing microcanonical simulations with various energy densities. From the data reported in Fig. 4, one can notice a divergence for $T \rightarrow 0$ of the type $\kappa \approx \exp (\alpha / T)$ with $\alpha \approx 1.2$. An even more convincing evidence of this behavior is provided by the temperature dependence of the average escape time (see the triangles in Fig. 4) with an exponent $\alpha \approx 2$. The latter behavior can be explained by assuming that the jumps are the results of activation processes. Accordingly, the probability of their occurrence is proportional to $\exp (-\Delta V / T)$, where $\Delta V$ is the barrier height and the Boltzmann constant is fixed equal to 1 (as implicitly done throughout the Letter). Since $\Delta V=2$, the whole interpretation is consistent. Moreover, in the absence of jumps, the dependence of the conductivity on the length should be the same as in FPU systems, i.e., $\kappa \approx N^{2 / 5}$. Therefore, a low-frequency mode traveling along the chain should experience a conductivity of the order of $\bar{N}^{2 / 5}$, where $\bar{N}$ is the average separation between jumps. Under the assumption of a uniform distribution of phase jumps, their spatial separation is of the same order of their time separation, so that we can expect that $\kappa \approx \exp [2 \Delta V /(5 T)]$. On the one hand, this heuristic argument explains why and how such jumps contribute to a normal transport. On the other hand, the numerical disagreement between the observed and the expected values of the exponent $\alpha$ (1.2 vs 0.8$)$ indicates

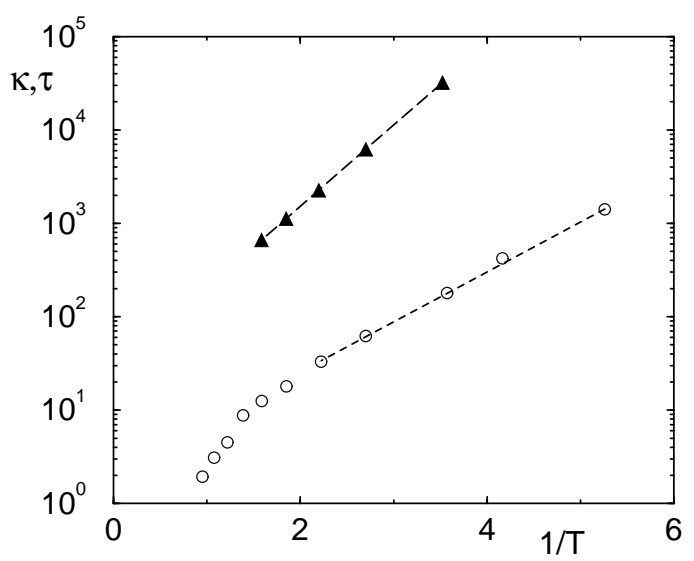

FIG. 4. Thermal conductivity $\kappa$ (labels on the left axis) versus the inverse temperature $1 / T$ in the rotator model (open circles). Triangles correspond to the average time separation between consecutive phase jumps (labels on the right axis) in the same system. 
that our analysis needs refinements. In fact, we should, e.g., notice that, in the low-energy limit, nonlinearities become negligible, implying that deviations from the asymptotic law $\kappa \approx N^{2 / 5}$ should become relevant.

(E) Further checks. - In order to test the conjecture that jumps are responsible for a normal heat transport, we have decided to investigate some other models. First, we have considered a double-well potential $V(x)=-x^{2} / 2+$ $x^{4} / 4$ (the same as in FPU with a different sign for the harmonic term). The results of the direct simulations are reported in Fig. 1 (see triangles) for a temperature corresponding again to a quarter of the barrier height. The finiteness of the conductivity and its numerical value is confirmed by the computation of $\kappa$ through the GreenKubo formula (see the light-grey shaded region).

Finally, we have considered an asymmetric version of the rotator model, namely, $V(x)=A-\cos (x)+$ $0.4 \sin (2 x)$, where $A$ is fixed in such a way that the minimum of the potential energy is zero, and the temperature again corresponds to one quarter of the barrier height. In this case too, the conductivity is finite, confirming our empirical idea that the jumps are responsible for breaking the coherence of the energy flux and, in turn, for the finite conductivity.

In conclusion, in this Letter we have reported about the first evidence of normal heat transport in 1D systems with momentum conservation. Such a behavior appears to be connected with jumps between neighboring potential valleys. From the dynamical point of view, it is natural to ask what are the peculiar properties of such jumps that make them so different from other types of nonlinear fluctuations that may occur in the single-well type of potentials. The only clearly distinctive feature that we have found is the "hyperbolic" type of behavior in the vicinity of a maximum of the potential which has to be confronted with the typical "elliptic" character of the oscillations around the minima. We hope to be able to understand in the future whether this is truly the reason for the difference in FPU and rotator systems.
We thank S. Lepri for several profitable discussions. One of us (A. P.) thanks Gat Omri for some remarks about our last comments. ISI in Torino is acknowledged for the kind hospitality during the Workshop on "Complexity and Chaos" 1998, where part of this work was performed.

[1] Throughout the paper we shall always assume $m_{i}=1$ unless otherwise stated.

[2] Z. Rieder, J. L. Lebowitz, and E. Lieb, J. Math. Phys. (N.Y.) 8, 1073 (1967).

[3] J. B. Keller, G. C. Papanicolau, and J. Weilenmann, Commun. Pure Appl. Math. XXXII, 583 (1978).

[4] G. Casati, J. Ford, F. Vivaldi, and W. M. Visscher, Phys. Rev. Lett. 52, 1861 (1984).

[5] T. Prosen and M. Robnik, J. Phys. A 25, 3449 (1992).

[6] S. Lepri, R. Livi, and A. Politi, Phys. Rev. Lett. 78, 1896 (1997); Physica (Amsterdam) 119D, 140 (1998).

[7] The lattice spacing is assumed equal to 1 without loss of generality.

[8] Y. Pomeau and R. Résibois, Phys. Rep. 19C, 63 (1975).

[9] S. Lepri, R. Livi, and A. Politi, Europhys. Lett. 43, 271 (1998).

[10] T. Hatano, Phys. Rev. E 59, 1 (1999); M. Vassalli, Diploma Thesis, University of Florence, Italy, 1999.

[11] B. Hu, B.-W. Li, and H. Zhao, Phys. Rev. E 57, 2992 (1998).

[12] G. Benettin, L. Galgani, and A. Giorgilli, Il Nuovo Cimento B 89, 89 (1985).

[13] R. Livi, M. Pettini, S. Ruffo, and A. Vulpiani, J. Stat. Phys. 48, 539 (1987); D. Escande, H. Kantz, R. Livi, and S. Ruffo, J. Stat. Phys. 76, 605 (1994).

[14] L. Casetti, C. Clementi, and M. Pettini, Phys. Rev. E 54, 5969 (1996).

[15] S. Nosé, J. Chem. Phys. 81, 511 (1984); W. G. Hoover, Phys. Rev. A 31, 1695 (1985).

[16] Ph. Choquard, Helv. Phys. Acta 36, 415 (1963).

[17] R. Kubo, M. Toda, and N. Hashitsume, Statistical Physics II, Springer Series in Solid State Sciences Vol. 31 (Springer, Berlin, 1991).

[18] R. I. McLachlan and P. Atela, Nonlinearity 5, 541 (1992). 